\title{
Comparison of Clinical and Biochemical Markers of Hirsutism in Polycystic Ovary Syndrome - A Study from a Teaching Hospital in Sri Ramachandra Institute, Chennai, India
}

\author{
Nandhini Logaprabhu' ${ }^{1}$, Sarmishta Murugesan ${ }^{2}$ \\ 1,2 Department of Obstetrics \& Gynaecology, Sri Ramachandra Institute of \\ Higher Education \& Research, Chennai, Tamilnadu, India.
}

\section{ABSTRACT}

\section{BACKGROUND}

We wanted to analyse the clinical profile of polycystic ovarian syndrome (PCOS) women with history, examination and ultrasonogram and correlate hirsutism with biochemical markers as free testosterone, dehydro-epiandrosterone sulphate (DHEAS), sex hormone binding globulin (SHBG), free testosterone, DHEAS, and SHBG.

\section{METHODS}

This study is a prospective observational study conducted from 2011 to 2013 in the Department of Obstetrics and Gynaecology, Shree Balaji Medical College and Hospital, Chrompet in patients attending Gynaecology OPD. 100 women visiting the OPD were taken as control and 100 women were taken for PCOS study.

\section{RESULTS}

Hyperandrogenism was studied and all the biochemical markers were significantly higher in polycystic ovarian syndrome patients than in controls $(\mathrm{P}<0.0001)$. The highest AUC-ROC was found for bioavailable testosterone (0.852) followed by free androgen index (0.847) and free testosterone (0.837). Lower AUC-ROC was found for androstenedione, total testosterone and SHBG $(0.706,0.799$ and 0.76 , respectively). When free androgen index of 4.97 was taken as a cut off value, sensitivity was $71.4 \%$ and specificity was $85.2 \%$. A cut off of $0.78 \mathrm{nmol} / \mathrm{L}$ for bioavailable testosterone had even higher sensitivity of $75.9 \%$, but slightly lower specificity of $83.3 \%$. Bioavailable testosterone and free androgen index correlated significantly (all $\mathrm{P}<0.05$ ) with DHEAS, LH / FSH ratio, androstenedione and total testosterone. In addition, bioavailable testosterone, free androgen index, and free testosterone correlated significantly with follicle count, ovarian volume, and hirsutism scores.

\section{CONCLUSIONS}

White women have about $20 \%$ of excess of dehydro-epiandrosterone sulphate (DHEAS) and black women have $30 \%$ excess of dehydro-epiandrosterone sulphate (DHEAS) in those having poly cystic ovaries patients. There is an age-associated decline in DHEAS levels which is similar in both control and poly cystic ovaries women, regardless of the race which was seen in this study.

\section{KEY WORDS}

Free Testosterone, Dehydro-Epiandrosterone Sulphate (DHEAS), Sex Hormone Binding Globulin (SHBG)
Corresponding Author: Dr. Sarmishta Murugesan, No. 1, Somasundaram Street,

T. Nagar, Chennai - 600017, Tamilnadu, India.,

E-mail: sarmishta83@yahoo.co.in

DOI: $10.14260 / j e m d s / 2021 / 243$

How to Cite This Article:

Logaprabhu N, Murugesan S. Comparison of clinical and biochemical markers of hirsutism in polycystic ovary syndrome - a study from a teaching hospital in Sri Ramachandra Institute, Chennai, India. J Evolution Med Dent Sci 2021;10(16):11401144, DOI: $10.14260 /$ jemds/2021/243

Submission 24-11-2020,

Peer Review 19-02-2021,

Acceptance 26-02-2021,

Published 19-04-2021.

Copyright (C) 2021 Nandhini Logaprabhu et al. This is an open access article distributed under Creative Commons Attribution License [Attribution 4.0 International (CC BY 4.0)] 


\section{BACKGROUND}

Polycystic ovarian syndrome is a common and heterogenous disorder of women with reproductive disorder, characterised by chronic anovulation, and hyperandrogenism with complex pathophysiology. It is one of the commonest endocrine metabolic disorders affecting $6-10 \%$ of women in the reproductive age. ${ }^{1}$ Features of PCOS may manifest at any age ranging from childhood to later life. Hyperandrogenism is defined as biochemical and poor clinical manifestation of androgen excess. ${ }^{2}$ It is a hallmark of PCOS. Acne, hirsutism, androgenic alopecia and seborrhoea, are cutaneous manifestations of hyperandrogenism and accompanies PCOS. About one in ten women in reproductive period have hirsutism, and PCOS is the commonest manifestation of hyperandrogenism. ${ }^{3}$ It is defined as development of male type of hair distribution in female due to conversion of vellus hair to terminal hair due to excess androgens. In 1935, Stein and Leventhal reported amenorrhea to be present in seven women and along with anovulation, bilateral enlarged polycystic ovaries with thickened tunica that were treated by wedge resection. Among them Stein reported in his study that 75 women who underwent wedge resection and among them 90 $\%$ of whom responded to regular menses and $65 \%$ of them conceived. In 1935, Stein and Leventhal described the association of enlarged, sclerocystic ovaries with amenorrhea, infertility and hirsutism as polycystic ovary syndrome. Today polycystic ovaries are one of the most common endocrine disorders. They reported seven women who presented with amenorrhea, anovulation and bilateral enlarged polycystic ovaries with thickened tunica and were treated by wedge resection. Later on, Stein reported another 75 women who also underwent wedge resection, $90 \%$ of whom responded to regular menses and $65 \%$ of them conceived. It is a heterogenous clinicopathological syndrome, may present with asymptomatic patient with a single finding of polycystic ovarian morphology on ultrasonogram or with spectrum of symptoms such as menstrual cycle disturbance, hyperandrogenism, obesity, infertility, metabolic disturbances, singly or in combination. Finding of polycystic ovaries does not mean that there is PCOS, as polycystic ovaries are found in $20 \%$ of otherwise normal women. The current definition of polycystic ovaries according to the Rotterdam consensus meeting on Polycystic ovaries held in 2003. It defines the syndrome of PCOS as the presence of any two of the following three criteria; ultrasound appearance of polycystic ovaries. Menstrual disturbances-evidence of hyperandrogenism, acne, hirsutism etc. After other causes are ruled out, especially congenital adrenal hyperplasia. Irregular menstrual cycles where at least 4 cycles per year decreases the risk of endometrial cancer. PCOS is present in $4-10 \%$ of premenopausal cohort and $30 \%$ among women attending the infertility clinics. Out of all women who show polycystic ovaries on USG, 50 - $85 \%$ will have symptoms and signs of menstrual irregularities. Cross sections of anovulatory women at any time revels $75 \%$ have PCO. Androgen excess is seen primarily as hirsutism. Hirsutism present together with oligomenorrhea and polycystic ovaries with normal menstrual cycles. $5 \alpha$-reductase type 2 helps in the synthesis of dihydrotestosterone (DHT) from testosterone which is the primary androgen responsible for hair growth. Women with hirsutism have increased $5 \alpha$-reductase-activity in their hair follicles. The women with hirsutism had their clinical scoring done according to Ferriman-Gallwey-Score. Clinical expression of menstrual dysfunction; in $66 \%$ of cases the history of menstrual disturbance dates back to menarche. 30 $\%$ of the women have amenorrhoea, $90 \%$ have oligomenorrhoea, and $90 \%$ polymenorrhoea is less frequent, indicative of short a luteal phase which further indicates dysovulation and anovulation due to unopposed oestrogen action on the endometrium. Dysfunctional uterine bleedingatypical and uncommon presentation. Polycystic ovaries are marked by hyperandrogenism. Androgens are natural hormones in the female and form an integral part of the endocrine system. Androgens are C-19 steroids and the parent nucleus androstane. The primary androgen producing glands in the female are the adrenal and the ovaries. In the ovaries, androgens are produced in the thecal layer by the action of $\mathrm{LH}$ on LH receptors. Insulin also acts on the LH receptors and hence hyperinsulinemia is seen in polycystic ovaries, also increases androgen production in females via LH mediated pathway. Androgens produced in the theca cell layer are converted to oestrogens in the granulosa cells by the enzyme aromatase. Only $1 \%$ of androgens in the female plasma are free, the rest is either bound to SHBG or loosely bound to albumin. Reduction of Serum hormone binding globulin increases the amount of free hormone which is responsible for clinical manifestations of hyperandrogenism. Excess of androgen in the system itself suppresses Serum hormone binding globulin which in turn increases the free level, thus a vicious cycle is established and hyperandrogenism persist. The most potent androgen testosterone is $70 \%$ SHBG bound compared to $8 \%$ of DHEAS and androstenedione. Hence, a slight reduction of SHBG increases free testosterone more than the others and cause significant hyperandrogenism even though the total testosterone is either normal or marginally increased.

Purpose of the study was to analyse the clinical profile of polycystic ovarian syndrome (PCOS) women with history, examination and Ultrasonogram and to determine the correlation of clinical manifestation of hirsutism with biochemical markers as free testosterone, dehydroepiandrosterone sulphate (DHEAS), sex hormone binding globulin (SHBG), free testosterone, DHEAS, SHBG.

\section{METHODS}

This is a prospective observational study conducted from January 2011 to January 2013 in the Department of Obstetrics and Gynaecology, Shree Balaji Medical College and Hospital, Chromepet. 100 women attending OPD were taken as control and 100 women were taken for PCOS study.

\section{Inclusion Criteria}

Age group between 20 - 30 years. Women fulfilling the Rotterdam's criteria. The newest diagnostic guideline was made by The Androgen Excess and Polycystic Ovaries Society, in 2006; they claim the presence of hyperandrogenism and ovarian dysfunction (oligo / anovulation and / or polycystic ovaries). 


\section{Exclusion Criteria}

- Pregnant women.

- Women using oral contraceptive pills.

- Hypothyroidism.

- Hyperprolactinemia.

After obtaining the informed consent and explaining the type and the need of the study, history was taken and clinical examination was done.

\section{Data Collection}

Subjects selected underwent a detailed history taking; all their presenting complaints were enquired. Duration of hirsutism and the age of onset of hirsutism were asked. Androgen secreting tumours present with shorter duration of hirsutism. Longer duration of hirsutism leads to idiopathic hirsutism. Patient was asked for history of irregular cycles or amenorrhea as polycystic ovaries have menstrual irregularities. Some patients have history of infertility. In polycystic ovaries there is an elevated level of androgen which leads to anovulation and infertility. Some patients have weight gain due to insulin resistance leading to polycystic ovaries. The conversion of testosterone to dihydrotestosterone is favoured by peripheral fat. The drug history, leading to polycystic ovaries is noted including the duration of the drug taken and name of the drug.

- Family history: Presence of hirsutism in family.

- Clinical examination: After history taking, they were subjected to anthropometric evaluation. Height was calculated in $\mathrm{cm}$ and weight was calculated in kilograms which were measured.

Body mass index was calculated with weight in $\mathrm{kg}$ / height in metre. Waist circumference was calculated in $\mathrm{cm}$ and hip circumference was calculated in $\mathrm{cm}$ which was measured for all subjects. Waist hip ratio was noted down for all the patients. Normal waist hip ratio is 0.8 . Insulin resistance is diagnosed with Waist-hip ratio greater than 0.8 . Hirsutism scoring was done according to modified Ferriman- Gallwey scoring system (a score of more than 8 suggests clinical hirsutism) skin-acne, seborrhoea, male pattern baldness, acanthosis nigricans (HAIR-AN syndrome), striae and thin skin (Cushing's syndrome). Other signs of virilisation were deep voice, hypertrichosis, acanthosis nigricans, male pattern balding, body habitus, increased muscle mass, clitoromegaly and breast atrophy. ${ }^{4}$ Cushing's syndrome which included buffalo hump and moon facies and features of milk secretion which indicates hyperprolactinemia were also looked for.

- Abdominal examination

- Pelvic examination

- USG pelvis

USG evidence of polycystic ovaries (according to Rotterdam's criteria). Those having 12 or more follicles with each in size of $2-9 \mathrm{~mm}$ and the volume of the ovary $>10 \mathrm{ml}$ in 1 or more ovaries were said to have polycystic ovaries. ${ }^{5}$ Ultrasound also showed the presence of adnexal masses and any uterine abnormalities. All women were subjected to blood test on day 3 of periods which involved measuring sex hormone binding globulin (SHBG), free testosterone and dehydroepiandrosterone sulphate (DHEAS). ${ }^{6}$ All the biochemical investigations were taken in the morning. The more sensitive indicator and an unbound hormone causing hirsutism is free testosterone. DHEAS is a direct measure of adrenal androgen activity. SHBG is a good measure of insulin resistance.

\section{Statistical Analysis}

P-value was calculated by chi-square test. The statistical software used was SPSS-16 (Statistical Package for the Software Solutions). Continuous variables were expressed in mean and standard deviation. Pearson's correlation was used for a combination of continuous and binary variables. In contrast, Independent t-tests examine are used to find significant differences between two group means.

\section{RESULTS}

In PCOS group, $36 \%$ were unmarried women and $64 \%$ were married women. The control group had $38 \%$ unmarried and $62 \%$ married women. The P-value was 0.836 , which is statistically insignificant. Table 2 shows the complaints of the PCOS study group. The major complaint being the menstrual irregularities with $50 \%$ and infertility being $48 \%$ and secondary infertility being $2 \%$. The correlation co-efficient for SHBG with BMI and waist-to-hip ratio (WHR) was calculated. The P-value for BMI was $<0.001$ and the P-value for weight height ratio was 0.005 which is statistically significant. The mean of BMI and WHR was 28.71 and 0.8253 respectively. The mean age for the PCOS was 25 years for control group was 23 years.

\begin{tabular}{|c|c|c|c|c|c|c|c|}
\hline \multirow{3}{*}{$\begin{array}{c}\text { Marital } \\
\text { Status }\end{array}$} & \multicolumn{4}{|c|}{ Group } & \multirow{2}{*}{\multicolumn{2}{|c|}{ Total }} & \multirow{3}{*}{ P-Value } \\
\hline & \multicolumn{2}{|c|}{ PCOS } & \multicolumn{2}{|c|}{ Control } & & & \\
\hline & $\mathbf{N}$ & $\%$ & $\mathbf{N}$ & $\%$ & $\mathbf{N}$ & $\%$ & \\
\hline Unmarried & 18 & 36.0 & 19 & 38.0 & 37 & 37.0 & \multirow{2}{*}{0.836} \\
\hline Married & 32 & 64.0 & 31 & 62.0 & 63 & 63.0 & \\
\hline Total & 50 & 100.0 & 50 & 100.0 & 100 & 100.0 & \\
\hline \multicolumn{8}{|c|}{ Table 1. Marital Status in All the Polycystic Groups } \\
\hline
\end{tabular}

\begin{tabular}{|ccc|}
\hline Complaints & Frequency & Percent \\
None & 25 & 50.0 \\
Infertility & 24 & 48.0 \\
Secondary infertility & 1 & 2.0 \\
Total & 50 & 100.0 \\
\hline Table 2. Major Complaints in the Polycystic Ovaries \\
\hline
\end{tabular}

\begin{tabular}{|ccc|}
\hline & Correlation & SHBG \\
\multirow{2}{*}{ BMI } & P-value & -0.543 \\
& $\mathrm{n}$ & $<0.001$ \\
& Correlation & 100 \\
\multirow{2}{*}{ WHR } & P-value & -0.281 \\
& $\mathrm{n}$ & 0.005 \\
\hline Table 3. Correlation Co-Efficient for SHBG with BMI and WHR & 100 \\
\hline
\end{tabular}

\begin{tabular}{|ccc|}
\hline Statistic & BMI & WHR \\
Number & 100 & 100 \\
Mean & 28.71 & 0.8253 \\
Standard deviation & 2.886 & 0.0571 \\
Minimum & 20 & 0.64 \\
Maximum & 36 & 0.97 \\
\hline \multicolumn{2}{|c|}{ Table 4. Descriptive Statistics } \\
\hline
\end{tabular}


The mean BMI of PCOS group was 29.16 and for the control group was 28.26. The mean WHR for the PCOS group was 0.833 and for control group was 0.818 . The mean free testosterone for the PCOS group was 2.611, for control group was 1.136. The mean DHEAS for the PCOS group was 7.722 for control group was 5.148. The mean SHBG for the PCOS group was 56.22 for control group was 48.56 .

\begin{tabular}{|c|c|c|c|c|c|}
\hline Variables & Group & $\mathbf{N}$ & Mean & Std. Deviation & P-Value \\
\hline \multirow{2}{*}{ Age (yrs.) } & PCOS & 50 & 25.04 & 4.257 & \multirow{2}{*}{0.034} \\
\hline & Control & 50 & 23.28 & 3.939 & \\
\hline \multirow{2}{*}{ BMI } & PCOS & 50 & 29.16 & 3.152 & \multirow{2}{*}{0.119} \\
\hline & Control & 50 & 28.26 & 2.546 & \\
\hline \multirow{2}{*}{ WHR } & PCOS & 50 & 0.833 & 0.0238 & \multirow{2}{*}{0.192} \\
\hline & Control & 50 & 0.818 & 0.0768 & \\
\hline \multirow{2}{*}{$\begin{array}{c}\mathrm{F} \\
\text { testosterone }\end{array}$} & PCOS & 50 & 2.611 & 2.113 & \multirow{2}{*}{$<0.001$} \\
\hline & Control & 50 & 1.136 & 0.599 & \\
\hline \multirow{2}{*}{ DHEAS } & PCOS & 50 & 7.722 & 2.542 & \multirow{2}{*}{$<0.001$} \\
\hline & Control & 50 & 5.148 & 2.438 & \\
\hline \multirow{2}{*}{ SHBG } & PCOS & 50 & 56.22 & 23.89 & \multirow{2}{*}{0.096} \\
\hline & Control & 50 & 48.56 & 21.57 & \\
\hline & $\begin{array}{r}\text { Table } 5 . \\
\text { and Co }\end{array}$ & 1 & $\begin{array}{l}\text { n Valu } \\
\text { oups w }\end{array}$ & $\begin{array}{l}\text { between PCOS } \\
\text { e Compared }\end{array}$ & \\
\hline
\end{tabular}

\section{DISCUSSION}

An important abnormality of hypothalamic-pituitary-ovarian system is inappropriate gonadotropin secretion. Although circulating levels of FSH in PCOS women are similar to normal women, induction aromatase enzyme and subsequent intrafollicular production of oestrogen seems to be distributed, which in turn negatively affects the ongoing follicular development as well as selection of dominant follicle. ${ }^{7}$ Kozloviene et al. at Lithuania concluded a study in which hormonal and clinical changes in women with hirsutism between the age group of 18 - 35 years in whom there was significantly lower levels of sex hormone binding globulin $(\mathrm{P}<$ 0.01 ), higher levels of free androgen index and higher levels of testosterone and dehydroepiandrosterone sulphate were found in females with hirsutism. Women with hirsutism had complaints of higher systolic and diastolic blood pressure, infertility, higher body mass index, increased skin greasiness, higher waist-hip ratio and larger waist and hip circumference. In a large series study of women diagnosed as having androgen excess by Hahn $\mathrm{S}$ and Kuehnel $\mathrm{W}$ et al. the clinical features observed were hirsutism (75\%), oligo-ovulation (88\%), infertility (30\%) and acne (14\%). The final diagnosis for these patients was polycystic ovary syndrome (PCOS) (82 \%); androgen-secreting neoplasms (0.2\%); idiopathic hirsutism (5\%); congenital adrenal hyperplasia (0.2\%); non-classical congenital adrenal hyperplasia (1.6\%); and hyperandrogenism with hirsutism and ovulation (7\%). Of these patients, $33 \%$ had elevation of both total and free testosterone, while $61 \%$ had an elevation of at least one of the testosterone fractions. Polycystic ovary syndrome is now considered a disorder of androgen excess, with $60-80 \%$ of patients having an elevation of at least one testosterone fraction, usually free testosterone. Diagnosis of PCOS is made after exclusion of other disorders which cause androgen excess. ${ }^{8}$ The syndrome of virilism (frontal balding, hirsutism, deepening of the voice, and acne) is almost always associated with excess testosterone. An uncommon masculinising ovarian tumour is named arrhenoblastoma. A consensus statement has released the guidelines for female androgen insufficiency. ${ }^{9}$ In this statement, the limitations of testosterone methods were recognized, and no cut-off level of testosterone was given. Rather, it was stated that the free testosterone levels should be at or below the lowest quartile of the reference interval for the reproductive age range of $20-40$ years. Analytical issues with the diagnosis of female androgen deficiency have been discussed and the existence of female androgen insufficiency continues to be strongly debated. Comparison of free testosterone methods, including use of the FAI, has demonstrated that the preferred methods for use in female androgen insufficiency are free testosterone determined either by equilibrium dialysis or by calculation using the law of mass action. Sullivan Nicolaides pathology (SNP) technical is a latter method which is technically used now and notes the methods for determination of free testosterone. Equilibrium dialysis (ED) is considered to be the 'gold standard' for determination of free testosterone. According to the recent studies which involve comparing the equilibrium dialysis methods with the free androgen index (FAI); immunoassay methods used by SNP for measurement of free testosterone; and calculations of free testosterone, have demonstrated that the calculation of free testosterone is the clinically preferred option. Data, comment and recommendations are included in the US Endocrine Society's position statement on measurement of testosterone. ${ }^{10} \mathrm{~A}$ brief synopsis of problems with free testosterone and FAI follows. Free testosterone estimates can be calculated according to the law of mass action using measured levels of testosterone, SHBG and albumin, together with assigned values for dissociation constants. In general, the calculated free testosterone estimates agree well with those determined by equilibrium dialysis-except in pregnancy. Currently there are no agreed dissociation constants for the binding of testosterone to either SHBG or albumin. In general, the dissociation constant of testosterone for SHBG is approximately $1 \mathrm{nmol}$, but values from 0.4 to $1.6 \mathrm{nmol}$ have been used. Variations between measured and calculated free testosterone can be attributed to the accuracy of methods of estimating testosterone and SHBG; and the value of dissociation constants for SHBG and albumin. The mean total testosterone, free testosterone, Androstenedione 4, DHEAS and body mass index were higher in women with polycystic ovaries than in control women. DHEAS levels were significantly lower in black women who were used as controls than white women who were used as controls, whereas fasting insulin and the body mass index were higher in black women who were used as controls. In controls and black women who had polycystic ovaries, the DHEAS levels did not correlate with BMI, waist-to-hip ratio or fasting insulin. Among the white women with polycystic ovaries, DHEAS levels correlated negatively with BMI and fasting insulin. DHEAS levels decreased similarly with age in control and polycystic women of either race. The prevalence of polycystic ovaries with increased DHEAS levels was $33.3 \%$ and $19.9 \%$, respectively, among black and white women with PCOS.

\section{CONCLUSIONS}

Hyperandrogenism was studied and all the biochemical markers were significantly higher in polycystic ovarian 
syndrome patients than in controls $(\mathrm{P}<0.0001)$. The highest AUC-ROC was found for bioavailable testosterone (0.852) followed by free androgen index (0.847) and free testosterone (0.837). Lower AUC-ROC was found for androstenedione, total testosterone and SHBG (0.706, 0.799 and 0.76, respectively). When free androgen index of 4.97 was taken as a cut off value, sensitivity was $71.4 \%$ and specificity was $85.2 \%$. A cut off of $0.78 \mathrm{nmol} / \mathrm{L}$ for bioavailable testosterone had even higher sensitivity of $75.9 \%$, but slightly lower specificity of $83.3 \%$. Bioavailable testosterone and free androgen index correlated significantly (all $\mathrm{P}<0.05$ ) with DHEAS, LH / FSH ratio, androstenedione and total testosterone. In addition, bioavailable testosterone, free androgen index, and free testosterone correlated significantly with follicle count, ovarian volume, and hirsutism scores. White women have about $20 \%$ of excess of dehydro- epiandrosterone sulphate (DHEAS) and black women have $30 \%$ excess of dehydroepiandrosterone sulphate (DHEAS) in those having poly cystic ovaries patients. There is an age-associated decline in DHEAS levels which are similar in both control and poly cystic ovaries women, regardless of the race which was seen in this study.

Data sharing statement provided by the authors is available with the full text of this article at jemds.com.

Financial or other competing interests: None.

Disclosure forms provided by the authors are available with the full text of this article at jemds.com.

\section{REFERENCES}

[1] Tchernof A, Labrie F. Dehydroepiandrosterone, obesity and cardiovascular disease risk: a review of human studies. Eur J Endocrinol 2004;151(1):1-14

[2] Krobath PD, Salek FS, Pittenger AL, et al. DHEA and DHEAS: a review. J Clin Pharmacol 1999;39(4):327-48.

[3] Imrich R. The role of neuroendocrine system in the pathogenesis of rheumatic diseases. Endocr Regul 2002;36(2):95-106.

[4] Thijs L, Fagard R., Forette F, et al. Are low dehydroepiandrosteronesulphate levels predictive for cardiovascular diseases? A review of prospective and retrospective studies. Acta Cardiol 2003;58(5):403-10.

[5] Chen CCG, Parker CR Jr. Adrenal androgens and the immune system. Semin Reprod Med 2004;22(4):369-77.

[6] Dharia S, Parke CR Jr. Adrenal androgens and aging. Semin Reprod Med 2004;22(4):361-8.

[7] Rosmond R. Androgen excess in women--a health hazard? Med Hypotheses 2006;67(2):229-34.

[8] Majewska MD, Demirgören S, Spivak CE, et al. The neurosteroid dehydroepiandrosterone sulfate is an allosteric antagonist of the GABAA receptor. Brain Res 1990;526(1):143-6.

[9] Charalampopoulos I, Alexaki VI, Tsatsanis C, et al. Neurosteroids as endogenous inhibitors of neuronal cell apoptosis in aging. Ann N Y Acad Sci 2006;1088:139-52.

[10] Campbell B. Adrenarche and the evolution of human life history. Am J Hum Biol 2006;18(5):569-89. 